\title{
Frontotemporal Lobar Degeneration and MicroRNAs
}

\author{
Paola Piscopo ${ }^{1 *}$, Diego Albani ${ }^{2 *}$, Anna E. Castellano ${ }^{3}$, Gianluigi Forloni ${ }^{2}$ and \\ Annamaria Confaloni ${ }^{1}$
}

${ }^{1}$ Department of Neuroscience, Istituto Superiore di Sanità, Rome, Italy, ${ }^{2}$ Department of Neuroscience, Istituto di Ricerche Farmacologiche Mario Negri, Milano, Italy, ${ }^{3}$ Department of Neurology, Neuromed Institute, Pozzilli, Italy

Frontotemporal lobar degeneration (FTLD) includes a spectrum of disorders characterized by changes of personality and social behavior and, often, a gradual and progressive language dysfunction. In the last years, several efforts have been fulfilled in identifying both genetic mutations and pathological proteins associated with FTLD. The molecular bases undergoing the onset and progression of the disease remain still unknown. Recent literature prompts an involvement of RNA metabolism in FTLD, particularly microRNAs (miRNAs). Dysregulation of miRNAs in several disorders, including neurodegenerative diseases, and increasing importance of circulating miRNAs in different pathologies has suggested to implement the study of their possible application as biological markers and new therapeutic targets; moreover, miRNA-based therapy is becoming a powerful tool to deepen the function of a gene, the mechanism of a disease, and validate therapeutic targets. Regarding FTLD, different studies showed

OPEN ACCESS

Edited by:

Isidro Ferrer,

University of Barcelona, Spain

Reviewed by:

Franc Llorens,

University Medical Center Göttingen,

Germany

Grazia Daniela Femminella,

University of Naples Federico II, Italy

*Correspondence:

Paola Piscopo

paola.piscopo@iss.it;

Diego Albani

diego.albani@marionegri.it

Received: 28 October 2015 Accepted: 21 January 2016 Published: 09 February 2016

Citation:

Piscopo P, Albani D, Castellano AE,

Forloni $G$ and Confaloni $A$ (2016)

Frontotemporal Lobar Degeneration and MicroRNAs.

Front. Aging Neurosci. 8:17. doi: 10.3389/fnagi.2016.00017 that miRNAs are playing an important role. For example, several reports have evaluated miRNA regulation of the progranulin gene suggesting that it is under their control, as described for miR-29b, miR-107, and miR-659. More recently, it has been demonstrated that TMEM106B gene, which protein is elevated in FTLD-TDP brains, is repressed by miR-132/212 cluster; this post-transcriptional mechanism increases intracellular levels of progranulin, affecting its pathways. These findings if confirmed could suggest that these microRNAs have a role as potential targets for some related-FTLD genes. In this review, we focus on the emerging roles of the miRNAs in the pathogenesis of FTLD.

Keywords: miRNA, frontotemporal lobar degeneration, progranulin, TDP43, social behavioral deficits

\section{INTRODUCTION}

Frontotemporal lobar degeneration (FTLD) is a pathological process that represents one of the main causes of dementia (Cardarelli et al., 2010), after Alzheimer's Disease (AD), accounting for $5-10 \%$ of all dementias and is characterized by atrophy in the frontal and temporal lobes of the brain (Seltman and Matthews, 2012). At clinical level, FTLD patients manifest personality and social behavior changes and, often gradual and progressive language dysfunction (McKhann et al., 2001). Approximately 15\% of patients also develop symptoms of motor neuron dysfunction. Pathologically, around $40 \%$ of FTLD patients present with inclusions of hyperphosphorylated microtubule-associated protein tau (FTLD-tau; Morris et al., 2001), whereas the most FTLD patients shows ubiquitin-positive inclusions (FTLD-U) constituted mainly by TAR DNA-binding protein 43 (TDP-43; Neumann et al., 2006). Genetically, several genes are described in association 
to the disease, including microtubule associated protein tau (MAPT), progranulin (GRN), and C9ORF72. Rare mutations were also found in genes coding charged multivesicular body protein $2 \mathrm{~B}(C H M P 2 B)$, the valosin-containing protein $(V C P)$, TAR DNA-binding protein (TARDBP), and FUS (Sieben et al., 2012). The genetic heterogeneity is further complicated by possible different clinical manifestations within a single mutation and even within individual families, suggesting the involvement of post-transcriptional regulation mechanisms such as microRNAs (miRNAs).

\section{miRNA BIOGENESIS AND FUNCTIONS}

Aberrant RNA processing can cause or exacerbate neurodegenerative diseases via many mechanisms. Less than $5 \%$ of total cellular RNA is messenger RNA coding for proteins, and the other $95 \%$ is non-coding RNAs that has been shown to have a profound impact on gene expression regulation and also on other neurochemical processes, and to be implicated as complexity multipliers in both normal and abnormal conditions of the human central nervous system (Nelson and Keller, 2007; Peterson et al., 2009; Rapoport and Nelson, 2011). miRNAs are members of non-coding RNAs and silence mRNA molecules via base-pairing with their complementary sequences (Fire et al., 1998). miRNAs are involved in all development and pathologic processes targeting protein-coding transcripts mainly in the $3^{\prime}$ UTR (Lytle et al., 2007). The biogenesis of miRNAs is controlled at temporal and spatial level. miRNAs are composed of $\sim 22$ nucleotides and produced by two RNase III proteins: Drosha and Dicer. Indeed, after the transcription, primary miRNAs (pri-miRNA) undergo to following steps: Drosha cleaves at the base of a stem to generate $\sim 60-100 \mathrm{nt}$ hairpin precursor miRNAs (pre-miRNA; Lee et al., 2003; Denli et al., 2004); then, pre-miRNA is carried into the cytoplasm by exportin 5 (Exp5) and once in the cytoplasm, it is processed by Dicer, arising a mature duplex of miRNAs long about 22 bp (Cullen, 2004; Winter and Diederichs, 2011).

\section{FTLD Pathogenically-Related Proteins and miRNA Biogenesis}

TDP-43, one of FTLD pathogenically-related proteins, binds both DNA and RNA and has multiple functions in mRNA metabolism contributing to transcriptional repression, pre-mRNA splicing and translational regulation (Buratti et al., 2001; Wang et al., 2008a; Da Cruz and Cleveland, 2011). Interestingly, TDP-43 has been found in a macromolecular complex containing Drosha and Dicer (Gregory et al., 2004; Kawahara and Mieda-Sato, 2012). In addition, TDP-43 binds directly to a subset of pri-miRNAs to promoting the production of pre-miRNAs (Kawahara and Mieda-Sato, 2012). FUS, another FTLD pathogenically-related protein, is a highly conserved RNA/DNA binding protein, as well. It participates to regulation of gene expression and RNA processing to the DNA damage response (Wang et al., 2013). FUS was also found in the macromolecular complex containing Drosha (Kawahara and Mieda-Sato, 2012) and binding to primiRNAs for miRNA processing (Morlando et al., 2012).

\section{miRNAs IN THE NERVOUS SYSTEM}

miRNAs are abundant in the nervous system and show a brainspecific expression profile correlated to the expression of their targets. In fact, as suggested by several microarray studies, the brain expresses a large number of miRNAs that participate in nervous system physiology (Lagos-Quintana et al., 2002; Miska et al., 2004; Lim et al., 2005; Manakov et al., 2009). In particular, they have key roles in the regulation of different biological functions, as important mediators of plasticity and neurogenesis, in which they channelize cellular physiology toward neuronal differentiation. Moreover, they regulate the proliferation and selfrenewal of neural stem cells indirectly influencing neurogenesis (Stappert et al., 2015). miRNAs are dysregulated in several neurodegenerative diseases that share a final common pathway of neuronal cell death. Neurodegeneration includes complex pathogenic mechanisms; above all, aging is the main risk factor, but other common characteristics exist among these pathologies, such as neuroinflammation, protein aggregation, and mitochondrial dysfunction. Profiling analysis of miRNA expression in several nervous system disorders identified signatures correlated with the diagnosis, progression and prognosis of diseases, and treatment monitoring (Shafi et al., 2010; Langbaum et al., 2013). The role of specific miRNAs in a disease has been established in just a few cases and most of the mechanistic data originates from invertebrate model systems. In any case, the importance of miRNAs in neurodegenerative disorders and their emerging role in AD, Parkinson's disease, Huntington's disease, and Amyotrophic lateral sclerosis is becoming more and more evident. (Kim et al., 2007; Johnson et al., 2008; Wang et al., 2008b; Nunez-Iglesias et al., 2010; Lukiw et al., 2012; Grasso et al., 2015). In AD patients, for example, profiling studies highlighted dramatic changes in several miRNAs (i.e., miR-29 cluster, miR-107, miR-9, miR-125b, and miR-128; Delay et al., 2012). Although these alterations under pathological conditions should be interpreted with caution, they support the idea that dysregulation of miRNAs is a common mechanism in neurodegenerative diseases. Consequently, the study of miRNAs is a novel tool to understanding these diseases and possibly find therapeutic targets. For a complete coverage of the roles of miRNAs in other neurodegenerative diseases, we suggest some recent review articles (Gascon and Gao, 2012; Maciotta et al., 2013; Grasso et al., 2014; Femminella et al., 2015).

\section{miRNAs AND FTLD}

In the last years, several studies have been addressed to understand the role of miRNAs in the pathophysiology of FTLD. Two approaches are frequently used to study the involvement of miRNAs in the pathology. The first is based on a global approach of miRNA profiling with subsequent validation by RT-qPCR. The second approach is based on the analysis of miRNAs showing a direct connection with the disease.

\section{miRNAs Expression Profiling}

Kocerha and colleagues decribed an expression profiling study to identify miRNAs responsive to GRN haploinsufficiency. 
They analyzed the global miRNAs in the frontal cortex of 8 FTLD-TDP patients with GRN mutations and compared them to a population of 32 FTLD-TDP patients with no apparent genetic abnormalities (Kocerha et al., 2011). Using TaqMan Array Human MicroRNA, they identified 20 miRNAs differentially expressed $(P<0.05)$ in frontal cortex. Moreover, a validation study by RT-qPCR analyzes confirmed a differential expression for 9 of the 20 miRNAs in frontal cortex (miR33a*, let7i*, miR-516a-3p, miR-548b-5p, miR-548c-5p, miR-565, miR-571, miR-572, and miR-922). Since these data have not been functionally validated, the mechanism by which PGRN haploinsufficiency in FTLD patients leads to altered expression of these miRNAs is currently unclear and requires future studies. Moreover, little or nothing has been studied about the role of these miRNAs in brain or other neurodegenerative diseases. For example, miR-922 is described as a promoter of tau phosphorylation by down-regulating ubiquitin carboxy-terminal hydrolase L1 expression in AD (Zhao et al., 2014), but other studies are needed to explain their role on neurological disease pathogenesis.

An expression profiling study was also performed by RNA deep sequencing in miRNAs derived from well-characterized brain samples originated from autopsy series at the University of Kentucky AD Center (UK ADC; Hébert et al., 2013). They analyzed temporal neocortex gray matter samples of five FTLD cases, as well as non-demented controls $(n=2), \mathrm{AD}(n=5)$, dementia with Lewy bodies $(n=4)$, hippocampal sclerosis in aging $(n=4)$. From a total of 795 miRNAs expressed in the human brain, they identified 31 human miRNAs differently expressed. The validation study by miRNA RT-qPCR analysis reported a down-regulation of miR-132-3p in FTLD cases. A similar result was obtained by the same authors in $\mathrm{AD}$ patients, as well suggesting that this miRNA could have a more general function in neurodegeneration than a direct role in specific subtypes of pathology. Recently, this miRNA was found participating to a post-transcriptional mechanism that increases intracellular levels of PGRN, as reported below.

\section{miRNA-132, miR-29b, and miR-659 Regulate GRN Expression}

Progranulin (PGRN), encoded by the GRN gene, is a widely expressed protein involved in the regulation of cell growth and cell cycle progression (Bateman et al., 1990; He and Bateman, 2003). Mutations in the GRN gene are linked with FTLD (Baker et al., 2006). Recent findings suggest that GRN is under the control of miRNAs (Table 1).

\section{miR-132 and miR-132-3p Cluster}

miR-132 down-regulation was also recently observed in another cohort of FTLD-TDP brains (Baker et al., 2006), thus confirming the data from Hebert and colleagues (He and Bateman, 2003). This miRNA is a member of the miR-132 cluster (miR-132, miR132*, and miR-212). miR-132 and miR-212 target TMEM106B through two specific sites in the TMEM106B 3'UTR. Chen Plotkin and colleagues, having observed increased TMEM106B expression in FTLD-TDP, supposed a possible upstream role for miR-132/212. Effectively, they showed first of all that miR132 and miR-212 normally repress TMEM106B and then that they are decreased in FTLD-TDP. Moreover, an involvement of TMEM106B gene on GRN expression was also observed; in details, this gene was found to be linked to FTLD-TDP (Van Deerlin et al., 2010; Van der Zee et al., 2011) and TMEM106B risk genotypes correlated with decreased plasma progranulin levels (Finch et al., 2011). Overall, these results suggest a model in which decreased levels of miR-132/212 carry out to a higher TMEM106B expression leading to increased TMEM106B expression and to endosomal-lysosomal dysfunction, which may, in turn, further increase the levels of TMEM106B, and perturb PGRN pathways, thereby increasing the risk of developing FTLD-TDP (Chen-Plotkin et al., 2012).

Further evidence linking miR-132 to FTLD are the reports addressing its role to the regulate splicing process of the microtubule associated protein tau gene (Smith et al., 2011; Hebert et al., 2012) and an involvement in cognitive function in experimental models including neuronal/synaptic integrity and the brain's response to stressors (O'Neill, 2009; Mellios et al., 2011; Miller et al., 2012; Wanet et al., 2012; Shaltiel et al., 2013; Bicker et al., 2014).

\section{miR-107}

A miRNA assay (RIP-Chip) in neuron-like human cell lines showed that GRN is a strong target for miR-107 (Wang et al., 2010). This miRNA recognizes mainly sequence elements in the open reading frame rather than the $3^{\prime} \mathrm{UTR}$ of GRN mRNA; these sequences are highly conserved among vertebrate species. Wang and colleagues described a down-regulation of miR107 in a mouse model of traumatic brain injury, speculating that this miRNA plays a role in modulating neuronal repair and regeneration in the mammalian brain through molecular regulation of GRN and other mRNA targets. Moreover, they described that miR-107 has also been involved in AD. In addition, Noren Hooten et al. compared miRNA expression of blood mononuclear cells in young individuals related to old individuals by RT-qPCR analysis to test possible aging-related differences and found that miR-107 was significantly decreased in aged individuals (Noren Hooten et al., 2010). Taken to in consideration this evidence, it would be interesting to study the existence of a possible link between miR-107 expression and FTLD.

\section{miR-659}

Another miRNA regulating GRN expression is miR-659. Rademakers and colleagues described an allelic variant associated to FTLD (Rademakers et al., 2008) located in the 3'UTR of GRN corresponding to a predicted binding site for miR-659. They described that genotype TT for SNP rs5848 was associated to FTLD with a 3.2-fold increased risk to develop the pathology suggesting that a genetic variant in a miRNA binding-site can influence the risk for FTLD. They further demonstrate that miR659 can regulate GRN expression in vitro, with miR-659 binding more efficiently to the high risk $\mathrm{T}$ allele of rs5848. In order to evaluate the possibility that rs5848 variant is involved in GRN regulation in patients, they analyzed its expression in FTLD 
TABLE 1 | miRNAs involved in FTLD.

\begin{tabular}{|c|c|c|c|c|}
\hline Alterated miRNAs & Regulation & Target genes & Related pathway to FTD & References \\
\hline miR-132/212 Cluster & $\begin{array}{l}\text { Downregulated in FTLD-TDP } \\
\text { brains }\end{array}$ & TMEM106B & Regulating PGRN levels & $\begin{array}{l}\text { Chen-Plotkin et al., 2012; } \\
\text { Hébert et al., } 2013\end{array}$ \\
\hline miR-107 & $\begin{array}{l}\text { Downregulated in mice with } \\
\text { cortical traumatic brain injury }\end{array}$ & GRN & Regulating PGRN levels & Wang et al., 2010 \\
\hline miR-659 & $\begin{array}{l}\text { Genetic variant located in the } \\
3^{\prime}-U T R \text { of GRN within a } \\
\text { miR- } 659 \text { binding site }\end{array}$ & GRN & $\begin{array}{l}\text { Genetic variability in binding-site of } \\
\text { miR- } 659 \text { can increase the risk for } \\
\text { FTLD }\end{array}$ & Rademakers et al., 2008 \\
\hline miR-29b & - & GRN & Regulating PGRN levels & Jiao et al., 2010 \\
\hline \multirow[t]{2}{*}{ miR-9 and pri-miR-9-2 } & $\begin{array}{l}\text { Downregulated in patient } \\
\text { neurons }\end{array}$ & $\begin{array}{l}\text { Neuronal specification } \\
\text { genes }\end{array}$ & Neuronal toxicity & Zhang et al., 2013 \\
\hline & $\begin{array}{l}\text { Downregulated in TDP-43 } \\
\text { mutants of Drosophila }\end{array}$ & & & Li et al., 2013 \\
\hline miR-124 & $\begin{array}{l}\text { Downregulated in a FTD mouse } \\
\text { model }\end{array}$ & AMPAR & Regulating social behavior & Gascon et al., 2014 \\
\hline
\end{tabular}

patient brains revealing an association between GRN lower levels and the TT genotype. However, other studies are needed to verify a possible role of miR-659 in the brain and particularly in FTLD.

\section{miR-29b}

Another binding site in the $3^{\prime}$ UTR of GRN mRNA was identified for miR-29b. Indeed, Jiao and colleagues demonstrated that miR-29b interacts directly with the GRN $3^{\prime} \mathrm{UTR}$, regulating the GRN expression (Jiao et al., 2010). MiR-29b has a role in neurodevelopment and reach the highest level in adult mouse brain. Moreover, it is differentially expressed in $\mathrm{AD}$ patients (Hebert et al., 2008), even if to date we do not have evidence about a regulatory role of this miRNA in FTLD patients. Thus, several miRNAs may contribute to the pathogenesis of FTLD linked to progranulin deficiency.

\section{miR-9 and TDP43}

As previously reported, TDP-43 has two RNA recognition motifs and is involved in several roles of RNA metabolism (Da Cruz and Cleveland, 2011; Lee et al., 2011). Among these, TDP43 has been associated to miRNA processing; miR-9 is one of these and is described as an evolutively conserved miRNA, brain-specific and implicated in some neurodegenerative diseases. A work published by Zhang and colleagues described a down-regulation of miR-9 in iPSC-derived neurons (iPSC: induced pluripotent stem cells) of FTD/ALS patients with TDP-43 mutations; they explained their results as an a effect of decreased TDP-43 expression revealed in these neurons (Zhang et al., 2013). Moreover, they found that levels of both pre-miR-9-2 and primiR-9-2 were also reduced, suggesting that miRNA decrease did not seem to be due to a defect in the miRNA-processing pathway.

Recently, a work on mutant TDP-43 showed an involvement in FTLD of Drosha protein, one of the most important miRNA processing molecules involved in the biogenesis of miRNAs. In this study Drosha increased in correlation with a TDP-43 activation in Neuro 2A cells, leading to hypothesize that a mutant TDP-43 in FTLD-U, associated with Drosha instability, can induce neuronal toxicity (Kim et al., 2015). Also in Drosophila,
TDP-43 seems to regulate miR-9a levels by interacting with pri-miR-9a and likely promoting its stability (Li et al., 2013).

\section{miR-124 and Social Behavioral Deficits}

miR-124 is one of most abundant miRNAs in the brain known to be involved in neuronal development. (Gao, 2010). Furthermore, it seems to have a role also in neurodegeneration and in regulating social behavior in FTLD models. Gascon and colleagues studied a new mouse model of FTLD exhibiting deficits in sociability (Gascon et al., 2014). They found that miR-124 is down-regulated in this model causing a dysregulation in AMPA receptor (AMPAR) composition and a selective impairment in sociability. Moreover, they examined miR-124 and AMPAR expression in subjects with FTLD, focusing on the frontal cortex in the subset of cases with bvFTD, whose clinical presentation is closest to the phenotypes observed in their mouse model. They found a decrease in miR-124 expression and a concomitant up-regulation of two AMPAR subunits in the frontal cortex of bvFTD patients compared with age-matched controls. Furthermore, they studied miR-124 and AMPARs in established iPSC lines derived from subjects with bvFTD (Almeida et al., 2012, 2013), in which the expression of miR-124 and AMPARs in 8-week-old neurons were reduced and some AMPAR subunit mRNAs upregulated.

Increasing evidences support the presence of common miRNAs differentially expressed in several neurodegenerative diseases. For example, miR-132, decreased in FTLD brains, is emerging as gradually down-regulated during $\mathrm{AD}$ at early, mid, and late stages of disease and showing its important role in the maintenance of brain integrity (Lau et al., 2014). Another miRNA regulated in both FTLD and AD is miR-107 that decreases in the temporal cortex of LOAD patients; in luciferase assays, it showed a functional interaction with $3^{\prime} \mathrm{UTR}$ region of BACE1, a protease involved in the cleavage of APP for the generation of amyloidbeta (Wang et al., 2008b). miR-107 also controls the expression of other proteins relevant to $\mathrm{AD}$ pathology, such as cofilin (Yao et al., 2010), an actin-binding protein that accumulates in 
cytoplasmic inclusions. Also miR-29b was found to be alterated in $\mathrm{AD}$ and interacting with BACE, as described by Hebert and colleagues (Hebert et al., 2008). Moreover, it was hypothesized that deregulation of miR-29b in the brain was associated to the increase of apoptotic markers in $\mathrm{AD}$, since miR-29b has been shown to target a family of pro-apoptotic regulators (Delay et al., 2012). Then, it could be conceivable the existence of common miRNAs pathways involved in the molecular mechanisms of some neurodegenerative diseases.

\section{FINAL REMARKS}

In the last years, miRNAs studies are becoming more and more powerful to understand molecular mechanisms of complex disorders, such as dementias because they can regulate in finemanner the pathways involved in the onset and progression of the disease and represent molecular tools as biomarkers and new therapeutic targets. Recent literature prompts that miRNAs could have an upstream and/or downstream role in the frontotemporal pathology to regulate a cascade of events leading to the neurodegeneration.

The studies mentioned in this mini-review provide interesting clues about miRNAs involvement in the pathology, and even if rather fragmentary, they could be further deepened by using high-throughput techniques such as small-RNAseq possibly not

\section{REFERENCES}

Almeida, S., Gascon, E., Tran, H., Chou, H. J., Gendron, T. F., Degroot, S., et al. (2013). Modeling key pathological features of frontotemporal dementia with C9ORF72 repeat expansion in iPSC-derived human neurons. Acta Neuropathol. 126, 385-399. doi: 10.1007/s00401-013-1149-y

Almeida, S., Zhang, Z., Coppola, G., Mao, W., Futai, K., Karydas, A., et al. (2012). Induced pluripotent stem cell models of progranulin-deficient frontotemporal dementia uncover specific reversible neuronal defects. Cell Rep. 2, 789-798. doi: 10.1016/j.celrep.2012.09.007

Baker, M., Mackenzie, I. R., Pickering-Brown, S. M., Gass, J., Rademakers, R., Lindholm, C., et al. (2006). Mutations in progranulin cause tau-negative frontotemporal dementia linked to chromosome 17. Nature 442, 916-919. doi: 10.1038 /nature05016

Bateman, A., Belcourt, D., Bennett, H., Lazure, C., and Solomon, S. (1990). Granulins, a novel class of peptide from leukocytes. Biochem. Biophys. Res. Commun. 173, 1161-1168. doi: 10.1016/S0006-291X(05)80908-8

Bicker, S., Lackinger, M., Weiß, K., and Schratt, G. (2014). MicroRNA-132, -134, and -138: a microRNA troika rules in neuronal dendrites. Cell. Mol. Life Sci. 71, 3987-4005. doi: 10.1007/s00018-014-1671-7

Buratti, E., Dork, T., Zuccato, E., Pagani, F., Romano, M., and Baralle, F. E. (2001). Nuclear factor TDP-43 and SR proteins promote in vitro and in vivo CFTR exon 9 skipping. EMBO J. 20, 1774-1784. doi: 10.1093/emboj/20.7.1774

Cardarelli, R., Kertesz, A., and Knebl, J. A. (2010). Frontotemporal dementia: a review for primary care physicians. Am. Fam. Physician 82, 1372-1377.

Chen-Plotkin, A. S., Unger, T. L., Gallagher, M. D., Bill, E., Kwong, L. K., VolpicelliDaley, L., et al. (2012). TMEM106B, the risk gene for frontotemporal dementia, is regulated by the microRNA-132/212 cluster and affects progranulin pathways. J. Neurosci. 32, 11213-11227. doi: 10.1523/JNEUROSCI.052112.2012

Cullen, B. R. (2004). Derivation and function of small interfering RNAs and microRNAs. Virus Res. 102, 3-9. doi: 10.1016/j.virusres.2004.01.009

Da Cruz, S., and Cleveland, D. W. (2011). Understanding the role of TDP-43 and FUS/TLS in ALS and beyond. Curr. Opin. Neurobiol. 21, 904-919. doi: 10.1016/j.conb.2011.05.029 only in patients, but also in cellular and animal models. Several advances have been performed on the developing of FTLD models, such as tau and TDP-43 transgenic mice reproducing some features of FTLD, such as reduced survival, fragmentation and insolubility of protein aggregates, gliosis, and neuronal loss. Moreover, iPSCs are likely to become a standard in the field of neurodegenerative diseases, complementing, but not replacing, genetic animal models (Ittner et al., 2015). Methodologies used for miRNAs in FTLD ranged from Northern blotting to microarrays, but further advances could arrive from small RNASeq sequencing useful to detect small RNAs from very low amounts of starting biological material and discovery novel miRNAs expressed in a specific pathology.

Despite last advances on molecular and pathological bases in FTLD, the pathology remains still orphan for disease-modifying therapies. The identification of disease modulating factors will in turn ameliorate the knowledge of FTLD molecular pathways opening, in the future, to new therapeutic strategies.

\section{AUTHOR CONTRIBUTIONS}

PP, DA: Substantial contribution to the conception or organization of the manuscript; AEC and GF: Drafting the work and revising it critically; and AC: Final approval of the version to be published.

Delay, C., Mandemakers, W., and Hébert, S. S. (2012). MicroRNAs in Alzheimer's disease. Neurobiol. Dis. 46, 285-290. doi: 10.1016/j.nbd.2012.01.003

Denli, A. M., Tops, B. B., Plasterk, R. H., Ketting, R. F., and Hannon, G. J. (2004). Processing of primary microRNAs by the microprocessor complex. Nature 432, 231-235. doi: 10.1038/nature03049

Femminella, G. D., Ferrara, N., and Rengo, G. (2015). The emerging role of microRNAs in Alzheimer's disease. Front. Physiol. 6:40. doi: 10.3389/fphys.2015.00040

Finch, N., Carrasquillo, M. M., Baker, M., Rutherford, N. J., Coppola, G., DejesusHernandez, M., et al. (2011). TMEM106B regulates progranulin levels and the penetrance of FTLD in GRN mutation carriers. Neurology 76, 467-474. doi: 10.1212/WNL.0b013e31820a0e3b

Fire, A., Xu, S., Montgomery, M. K., Kostas, S. A., Driver, S. E., and Mello, C. C. (1998). Potent and specific genetic interference by double-stranded RNA in Caenorhabditis elegans. Nature 391, 806-811.

Gao, F. B. (2010). Context-dependent functions of specific microRNAs in neuronal development. Neural Dev. 5:25. doi: 10.1186/1749-8104-5-25

Gascon, E., and Gao, F. B. (2012). Cause or effect: misregulation of microRNA pathways in neurodegeneration. Front. Neurosci. 6:48. doi: 10.3389/fnins.2012.00048

Gascon, E., Lynch, K., Ruan, H., Almeida, S., Verheyden, J. M., Seeley, W. W., et al. (2014). Alterations in microRNA-124 and AMPA receptors contribute to social behavioral deficits in frontotemporal dementia. Nat. Med. 20, 1444-1451. doi: $10.1038 / \mathrm{nm} .3717$

Grasso, M., Piscopo, P., Confaloni, A., and Denti, M. A. (2014). Circulating miRNAs as biomarkers for neurodegenerative disorders. Molecules 19, 6891-6910. doi: 10.3390/molecules19056891

Grasso, M., Piscopo, P., Crestini, A., Confaloni, A., and Denti, M. A. (2015). Circulating microRNAs in neurodegenerative diseases. EXS 106, 151-169. doi: 10.1007/978-3-0348-0955-9_7

Gregory, R. I., Yan, K. P., Amuthan, G., Chendrimada, T., Doratotaj, B., Cooch, N., et al. (2004). The microprocessor complex mediates the genesis of microRNAs. Nature 432, 235-240. doi: 10.1038/nature03120

Hebert, S. S., Horrè, K., Nicolaï, L., Papadopoulou, A. S., Mandemakers, W., Silahtaroglu, A. N., et al. (2008). Loss of microRNA cluster miR-29a/b-1 
in sporadic Alzheimer's disease correlates with increased BACE1/betasecretase expression. Proc. Natl. Acad. Sci. U.S.A. 105, 6415-1520. doi: 10.1073/pnas.0710263105

Hebert, S. S., Sergeant, N., and Buee, L. (2012). MicroRNAs and the regulation of tau metabolism. Int. J. Alzheimers Dis. 2012:406561. doi: 10.1155/2012/406561

Hébert, S. S., Wang, W. X., Zhu, Q., and Nelson, P. T. (2013). A study of small RNAs from cerebral neocortex of pathology-verified Alzheimer's disease, dementia with lewy bodies, hippocampal sclerosis, frontotemporal lobar dementia, and non-demented human controls. J. Alzheimers Dis. 35, 335-348. doi: $10.3233 /$ JAD-122350

He, Z., and Bateman, A. (2003). Progranulin (granulin-epithelin precursor, PC-cell-derived growth factor, acrogranin) mediates tissue repair and tumorigenesis. J. Mol. Med. 81, 600-612. doi: 10.1007/s00109-003-0474-3

Ittner, L. M., Halliday, G. M., Kril, J. J., Götz, J., Hodges, J. R., and Kiernan, M. C. (2015). FTD and ALS-translating mouse studies into clinical trials. Nat. Rev. Neurol. 11, 360-366. doi: 10.1038/nrneurol.2015.65

Jiao, J., Herl, L. D., Farese, R. V., and Gao, F. B. (2010). MicroRNA$29 \mathrm{~b}$ regulates the expression level of human progranulin, a secreted glycoprotein implicated in frontotemporal dementia. PLOS ONE 5:e10551. doi: 10.1371/journal.pone.0010551

Johnson, R., Zuccato, C., Belyaev, N. D., Guest, D. J., Cattaneo, E., and Buckley, N. J. (2008). A microRNA-based gene dysregulation pathway in Huntington's disease. Neurobiol. Dis. 29, 438-445. doi: 10.1016/j.nbd.2007.11.001

Kawahara, Y., and Mieda-Sato, A. (2012). TDP-43 promotes microRNA biogenesis as a component of the Drosha and Dicer complexes. Proc. Natl. Acad. Sci. U.S.A. 109, 3347-3352. doi: 10.1073/pnas.1112427109

Kim, J., Inoue, K., Ishii, J., Vanti, W. B., Voronov, S. V., Murchison, E., et al. (2007). A MicroRNA feedback circuit in midbrain dopamine neurons. Science 317, 1220-1224. doi: 10.1126/science.1140481

Kim, K. Y., Lee, H. W., Shim, Y. M., Mook-Jung, I., Jeon, G. S., and Sung, J. J. (2015). A phosphomimetic mutant TDP-43 (S409/410E) induces Drosha instability and cytotoxicity in Neuro 2A cells. Biochem. Biophys. Res. Commun. 464, 236-243. doi: 10.1016/j.bbrc.2015.06.125

Kocerha, J., Kouri, N., Baker, M., Finch, N., DeJesus-Hernandez, M., Gonzalez, J., et al. (2011). Altered microRNA expression in frontotemporal lobar degeneration with TDP-43 pathology caused by progranulin mutations. BMC Genomics 12:527. doi: 10.1186/1471-2164-12-527

Lagos-Quintana, M., Rauhut, R., Yalcin, A., Meyer, J., Lendeckel, W., and Tuschl, T. (2002). Identification of tissue-specific microRNAs from mouse. Curr. Biol. 12, 735-739. doi: 10.1016/S0960-9822(02)00809-6

Langbaum, J. B., Fleisher, A. S., Chen, K., Ayutyanont, N., Lopera, F., Quiroz, Y. T., et al. (2013). Ushering in the study and treatment of preclinical Alzheimer disease. Nat. Rev. Neurol. 9, 371-381. doi: 10.1038/nrneurol.2013.107

Lau, P., Frigerio, C. S., and De Strooper, B. (2014). Variance in the identification of microRNAs deregulated in Alzheimer's disease and possible role of lincRNAs in the pathology: the need of larger datasets. Ageing Res. Rev. 17, 43-53. doi: 10.1016/j.arr.2014.02.006

Lee, E. B., Lee, V. M., and Trojanowski, J. Q. (2011). Gains or losses: molecular mechanisms of TDP43-mediated neurodegeneration. Nat. Rev. Neurosci. 13, 38-50. doi: 10.1038/nrn3121

Lee, Y., Ahn, C., Han, J., Choi, H., Kim, J., Yim, J., et al. (2003). The nuclear RNase III Drosha initiates microRNA processing. Nature 425, 415-419. doi: 10.1038/nature01957

Li, Z., Lu, Y., Xu, X. L., and Gao, F. B. (2013). The FTD/ALS-associated RNAbinding protein TDP-43 regulates the robustness of neuronal specification through microRNA-9a in Drosophila. Hum. Mol. Genet. 22, 218-225. doi: $10.1093 / \mathrm{hmg} / \mathrm{dds} 420$

Lim, L. P., Lau, N. C., Garrett-Engele, P., Grimson, A., Schelter, J. M., Castle, J., et al. (2005). Microarray analysis shows that some microRNAs downregulate large numbers of target mRNAs. Nature 433, 769-773. doi: 10.1038 /nature 03315

Lukiw, W. J., Alexandrov, P. N., and Zhao, Y. (2012). Spreading of Alzheimer's disease inflammatory signaling through soluble micro-RNA. Neuroreport 23, 621-626. doi: 10.1097/WNR.0b013e32835542b0

Lytle, J. R., Yario, T. A., and Steitz, J. A. (2007). Target mRNAs are repressed as efficiently by microRNA-binding sites in the $5^{\prime}$ UTR as in the 3 'UTR. Proc. Natl. Acad. Sci. U.S.A. 104, 9667-9672. doi: 10.1073/pnas.0703820104
Maciotta, S., Meregalli, M., and Torrente, Y. (2013). The involvement of microRNAs in neurodegenerative diseases. Front. Cell. Neurosci. 7:265. doi: $10.3389 /$ fncel.2013.00265

Manakov, S. A., Grant, S. G., and Enright, A. J. (2009). Reciprocal regulation of microRNA and mRNA profiles in neuronal development and synapse formation. BMC Genomics 10:419. doi: 10.1186/1471-2164-10-419

McKhann, G. M., Albert, M. S., Grossman, M., Miller, B., Dickson, D., and Trojanowski, J. Q. (2001). Work Group on frontotemporal dementia and pick's disease. clinical and pathological diagnosis of frontotemporal dementia: report of the Work Group on Frontotemporal Dementia and Pick's Disease. Arch. Neurol. 58, 1803-1809.

Mellios, N., Sugihara, H., Castro, J., Banerjee, A., Le, C., Kumar, A., et al. (2011). miR-132, an experience-dependent microRNA, is essential for visual cortex plasticity. Nat. Neurosci. 14, 1240-1242. doi: 10.1038/nn.2909

Miller, B. H., Zeier, Z., Xi, L., Lanz, T. A., Deng, S., Strathmann, J., et al. (2012). MicroRNA-132 dysregulation in schizophrenia has implications for both neurodevelopment and adult brain function. Proc. Natl. Acad. Sci. U.S.A. 109, 3125-3130. doi: 10.1073/pnas.1113793109

Miska, E. A., Alvarez-Saavedra, E., Townsend, M., Yoshii, A., Sestan, N., Rakic, P., et al. (2004). Microarray analysis of microRNA expression in the developing mammalian brain. Genome Biol. 5:R68 doi: 10.1186/gb-2004-5-9-r68

Morlando, M., Dini Modigliani, S., Torrelli, G., Rosa, A., Di Carlo, V., Caffarelli, E., et al. (2012). FUS stimulates microRNA biogenesis by facilitating co-transcriptional Drosha recruitment. EMBO J. 31, 4502-4510. doi: 10.1038/emboj.2012.319

Morris, H. R., Khan, M. N., Janssen, J. C., Brown, J. M., Perez-Tur, J., Baker, M., et al. (2001). The genetic and pathological classification of familial frontotemporal dementia. Arch. Neurol. 58, 1813-1816.

Nelson, P. T., and Keller, J. N. (2007). RNA in brain disease: no longer just "the messenger in the middle." J. Neuropathol. Exp. Neurol. 66, 461-468. doi: 10.1097/01.jnen.0000240474.27791.f3

Neumann, M., Sampathu, D. M., Kwong, L. K., Truax, A. C., Micsenyi, M. C., Chou, T. T., et al. (2006). Ubiquitinated TDP-43 in frontotemporal lobar degeneration and amyotrophic lateral sclerosis. Science 314, 130-133. doi: 10.1126/science. 1134108

Noren Hooten, N., Abdelmohsen, K., Gorospe, M., Ejiogu, N., Zonderman, A. B., and Evans, M. K. (2010). MicroRNA expression patterns reveal differential expression of target genes with age. PLOS ONE 5:e10724. doi: 10.1371/journal.pone.0010724

Nunez-Iglesias, J., Liu, C. C., Morgan, T. E., Finch, C. E., and Zhou, X. J. (2010). Joint genome-wide profiling of miRNA and mRNA expression in Alzheimer's disease cortex reveals altered miRNA regulation. PLOS ONE 5:e8898. doi: 10.1371/journal.pone.0008898

O'Neill, L. A. (2009). Boosting the brain's ability to block inflammation via microRNA-132. Immunity 31, 854-855. doi: 10.1016/j.immuni.2009.11.004

Peterson, K. J., Dietrich, M. R., and McPeek, M. A. (2009). MicroRNAs and metazoan macroevolution: insights into canalization, complexity, and the Cambrian explosion. Bioessays 31, 736-747. doi: 10.1002/bies.200900033

Rademakers, R., Eriksen, J. L., Baker, M., Robinson, T., Ahmed, Z., Lincoln, S. J., et al. (2008). Common variation in the miR-659 binding-site of GRN is a major risk factor for TDP43-positive frontotemporal dementia. Hum. Mol. Genet. 17, 3631-3642. doi: 10.1093/hmg/ddn257

Rapoport, S. I., and Nelson, P. T. (2011). Biomarkers and evolution in Alzheimer disease. Prog. Neurobiol. 95, 510-513. doi: 10.1016/j.pneurobio.2011.07.006

Seltman, R. E., and Matthews, B. R. (2012). Frontotemporal lobar degeneration: epidemiology, pathology, diagnosis and management. CNS Drugs 26, 841-870. doi: 10.2165/11640070-000000000-00000

Shafi, G., Aliya, N., and Munshi, A. (2010). Micro RNA signatures in neurological disorders. Can. J. Neurol. Sci. 37, 177-185. doi: 10.1017/S03171671000 09902

Shaltiel, G., Hanan, M., Wolf, Y., Barbash, S., Kovalev, E., Shoham, S., et al. (2013). Hippocampal microRNA-132 mediates stress-inducible cognitive deficits through its acetyl-cholinesterase target. Brain Struct. Funct. 218, 59-72. doi: 10.1007/s00429-011-0376-z

Sieben, A., Van Langenhove, T., Engelborghs, S., Martin, J. J., Boon, P., Cras, P., et al. (2012). The genetics and neuropathology of frontotemporal lobar degeneration. Acta Neuropathol. 124, 353-372. doi: 10.1007/s00401-012-1029-x 
Smith, P. Y., Delay, C., Girard, J., Papon, M. A., Planel, E., Sergeant, N., et al. (2011). MicroRNA-132 loss is associated with tau exon 10 inclusion in progressive supranuclear palsy. Hum. Mol. Genet. 20, 4016-4024. doi: 10.1093/hmg/ ddr330

Stappert, L., Roese-Koerner, B., and Brüstle, O. (2015). The role of microRNAs in human neural stem cells, neuronal differentiation and subtype specification. Cell Tissue Res. 359, 47-64. doi: 10.1007/s00441-014-1981-y

Van Deerlin, V. M., Sleiman, P. M., Martinez-Lage, M., Chen-Plotkin, A., Wang, L. S., Graff-Radford, N. R., et al. (2010). Common variants at 7p21 are associated with frontotemporal lobar degeneration with TDP-43 inclusions. Nat. Genet. 42, 234-239. doi: 10.1038/ng.536

Van der Zee, J., Van Langenhove, T., Kleinberger, G., Sleegers, K., Engelborghs, S., Vandenberghe, R., et al. (2011). TMEM106B is associated with frontotemporal lobar degeneration in a clinically diagnosed patient cohort. Brain 134, 808-815. doi: 10.1093/brain/awr007

Wanet, A., Tacheny, A., Arnould, T., and Renard, P. (2012). miR-212/ 132 expression and functions: within and beyond the neuronal compartment. Nucleic Acids Res. 40, 4742-4753. doi: 10.1093/nar/gks151

Wang, I. F., Wu, L. S., Chang, H. Y., and Shen, C. K. (2008a). TDP-43, the signature protein of FTLD-U, is a neuronal activity-responsive factor. J. Neurochem. 105, 797-806.

Wang, W. X., Rajeev, B. W., Stromberg, A. J., Ren, N., Tang, G., Huang, Q., et al. (2008b). The expression of microRNA miR-107 decreases early in Alzheimer's disease and may accelerate disease progression through regulation of beta-site amyloid precursor protein-cleaving enzyme 1. J. Neurosci. 28, 1213-1223. doi: 10.1523/JNEUROSCI.5065-07.2008

Wang, W. X., Wilfred, B. R., Madathil, S. K., Tang, G., Hu, Y., Dimayuga, J., et al. (2010). miR-107 regulates granulin/progranulin with implications for traumatic brain injury and neurodegenerative disease. Am. J. Pathol. 177, 334-345. doi: 10.2353/ajpath.2010.091202
Wang, W. Y., Pan, L., Su, S. C., Quinn, E. J., Sasaki, M., Jimenez, J. C., et al. (2013) Interaction of FUS and HDAC1 regulates DNA damage response and repair in neurons. Nat Neurosci. 16, 1383-1391. doi: 10.1038/nn.3514

Winter, J., and Diederichs, S. (2011). MicroRNA Northern blotting, precursor cloning, and Ago2-improved RNA interference. Methods Mol. Biol. 676, 85-100. doi: 10.1007/978-1-60761-863-8_7

Yao, J., Hennessey, T., Flynt, A., Lai, E., Beal, M. F., and Lin, M. T. (2010) MicroRNA-related cofilin abnormality in Alzheimer's disease. PLoS ONE 5:e15546. doi: 10.1371/journal.pone.0015546

Zhang, Z., Almeida, S., Lu, Y., Nishimura, A. L., Peng, L., Sun, D., et al. (2013). Downregulation of microRNA-9 in iPSC-derived neurons of FTD/ALS patients with TDP-43 mutations. PLoS ONE 8:e76055. doi: 10.1371/journal.pone.0076055

Zhao, Z. B., Wu, L., Xiong, R., Wang, L. L., Zhang, B., Wang, C., et al. (2014). MicroRNA-922 promotes tau phosphorylation by downregulating ubiquitin carboxy-terminal hydrolase L1 (UCHL1) expression in the pathogenesis of Alzheimer's disease. Neuroscience 275, 232-237. doi: 10.1016/j.neuroscience.2014.06.013

Conflict of Interest Statement: The authors declare that the research was conducted in the absence of any commercial or financial relationships that could be construed as a potential conflict of interest.

Copyright (C) 2016 Piscopo, Albani, Castellano, Forloni and Confaloni. This is an open-access article distributed under the terms of the Creative Commons Attribution License (CC BY). The use, distribution or reproduction in other forums is permitted, provided the original author(s) or licensor are credited and that the original publication in this journal is cited, in accordance with accepted academic practice. No use, distribution or reproduction is permitted which does not comply with these terms. 